Punjab University Journal of Mathematics (2021),53(6),387-408

https://doi.org/1.52280/pujm.2021.530603

\title{
Mathematical Model for Single and Multiple Object Extraction
}

\author{
Amna Shujahuddin ${ }^{1}$, Muhammad Salim Khan ${ }^{2}$, Haider $\mathrm{Ali}^{3}$ \\ ${ }^{1,3}$ Department of Mathematics, University of Peshawar, Pakistan. \\ ${ }^{2}$ Department of Basic Sciences. \\ University of Engineering and Technology, Peshawar, Pakistan. \\ Email: 1,2,3 amnashujjahuddin@gmail.com, salimgul.433@gmail.com, \\ dr.haider@uop.edu.pk \\ Corresponding author: salimgul.433@gmail.com
}

Received: 21 January, 2021 / Accepted: 14 June, 2021 / Published online: 25 June, 2021

\begin{abstract}
In the image processing, noise is referred to as the visual distortion. This undesirable by-product may be captured in an image due to unpreventable assorted reasons. The interference of natural phenomena and technical problem, such as small sensor size, long exposure time, low ISO, shadow noise etc., can pollute image. The presence of noise images affects image processing outputs that include segmentation. Segmentation for noisy images is the major concern. To tackle this issue, we propose a modernistic model that is able neutralize the negative effects of outlier using the characteristic of kernel function by different approaches such as linear approach and quadratic approach for global segmentation. Moreover the weight function is used for local segmentation of noisy images. Comparing with classical models, the proposed technique shows robust performance. In comparison with the wellknown models such as Chan-Vese (CV) model, Yongfei Wu and Chuanjiang $\mathrm{He}(\mathrm{Wu}-\mathrm{He})$ model and Chunming $\mathrm{Li}(\mathrm{Li})$ model we conclude that performance of our new model is much better.
\end{abstract}

AMS (MOS) Subject Classification Codes: 35S29; 40S70; 25 U09

Key Words: Active contours, kernel function, Variational approach, Image segmentation, de-noising.

\section{Introduction}

Image processing is the process which uses digitized image for analysis and manipulation to improve its quality. In image processing, image segmentation is the partitioning of an image into the constituent parts or objects for the purpose of analyzing and extracting meaningful information. Basically it is divided into two 
types i.e. global segmentation and local segmentation. In order to capture object of interest we use local segmentation while in global segmentation all objects are segmented. To extract valuable but small target object in image e.g brain tumor, blood clot, kidney stone, fracture in bone etc local segmentation [23, 2] helps for the purpose of exact calculation and results in the target of interest. Whereas global segmentation $[11,26,28,13,6,27,5]$ captures all details in image. For segmentation many approaches have been developed such as region growing methods, graph partitioning methods, semi-automatic segmentation, active contours without edge methods [24, 26, 27, 12], Clustering [14, 9].

Image segmentation is a tough and challenging task in various aspects [18], that is in presence of noise, intensity inhomogeneity, fog and haze, textures [5] and in other hard images. Noise is the natural phenomena but a crucial factor, when at some pixel the sudden change of intensity function occurs that change is called noise in an image. When image intensity information get disturbed almost completely then image become noisy image. Sometime images are corrupt by noise due to several inescapable reasons such as transfer image from on place to place, bad sensor quality and some natural occurring phenomena like atmosphere etc. On some occasion we add noise in a clear picture means in the pixels data of the image to check the performance of model. It is well understood that different type of noises such as additive noise salt and pepper, multiplicative noise or speckle may effect different models in different way. These noise have different effects and problems in images.

In noise images classical segmentation techniques which rely on edge detection fail to produce the desired segmentation results. To overcome this problem many models are devised with different approaches, the approach we use in this paper is variational approach $[19,4,8,1]$. The beauty of variational models is that these models are flexible for imposing geometrical constraints like regularity on the obtained solution. For global segmentation the most popular region based model is proposed by Chan-Vese named as "Active Contour Without Edges" [6] in which the contour of objects in image is the zero level set of the implicit function. This model segments the image of two-phase images having piecewise constant intensities, further this model is extended to the multi-phases [24] for which $\log _{2} n$ level sets are required. Further to improve this model for inhomogeneity Li et al. [13] proposed a model which is the modification of CV model which uses kernel function to control the path distance between $x^{t h}$ and $y^{t h}$ pixel. Later, to make it robust for noisy images Badshah and Chen [2] combined this model with a region term. Wu and $\mathrm{He}$ [25] also recently proposed a convex variational level set model based on the coefficient of variation [22], they proved that for any image the value of unique global minimizer $\phi$ for the energy functional lies in $-1 \leq \phi \leq 1$, and for any binary ideal image it is equal to -1 in the background and 1 in the object.

Many variational models are designed to segment a given image by ignoring the noise factor. Keeping in mind the limitations of the previously proposed model which are high sensitivity to noise and failure to isolate the desired object. One of the leading objective of this paper is to deal with the challenge of image segmentation by considering the effect of noise, by different approaches for both selectively and globally segmentation. In order to develop an efficient variational model which 
will be able to:

(1) Neutralize the negative effect of outlier.

(2) Perform selective segmentation.

(3) Segment noise images.

(4) Segment image with intensity inhomogeneity.

(5) Segment image with intensity inhomogeneity and noise together.

Overcome the negative effect of outlier in an image the concept of LBF model and CV model will be employed. The kernel function based on Gaussian kernel will be used which will help the level set function to be smooth. In order to enhance the performance we will use two-dimensional piecewise linear function and then quadratic function in the proposed model for gray-scale images. Further we will extend our work to segment only the desired object in the given image, for that the weight function [15] will be utilized. The remainder of the paper is structured as follows. Section 2 contains background work, Section 3 presents proposed model, Section 4 presents experimental results, while Section 5 includes some conclusive remarks.

\section{BACKGROUND}

The main topic in image processing is image segmentation under the influence of high noise. There are two main aspects of image processing i.e. creating visually clear perception of image for human and computer and other is to enhance the understanding of images for computer vision and processing tasks. The division of image into multiple similar segments on the basis of color, texture, intensities to extract information is called image segmentation. In image segmentation our ultimate aim is to distinguish some and all objects from the background. For image processing many popular models have been proposed such as CV model [6], Local Binary Fitting (LBF) model [16], Wu and He [25] model etc. The well known $\mathrm{CV}$ model utilizes the means to segment the image constant intensities, but this model fails when the intensities in an image is not constant, when image have noise and selective segmentation. To robust the CV model for inhomogeneity Li et al. developed LBF model, this model uses local image information so in a result this model doses not give promising outcome for the image having noise and high intensity inhomogeneity. Wu and He model is convex model but this model also have some limitations i.e. this model is not designed for noisy images and also this model is not capable of segmenting the target object. To identify the interface between two adjacent objects which owns the homogeneous texture in the given image these models face hurdles and fails. To overcome this issue C. L. Guyader and C. Gout .[15] proposed a model which utilizes the geometric constraints. This model localize the evolving curve $\phi$ but in presence of noise it fails to produce accurate result.

2.1. The Chan-Vese model (CV). Chan and Vese [6] proposed a novel model named as CV model which segments the objects in images having constant intensities.

Let $I_{0}$ be an image defined on $\Omega \subset \Re^{2}$ image domain and $\mathrm{C}$ be the moving curve of object. A region based model $[6,16,17]$ whose energy functional with length term is given by: 


$$
\begin{aligned}
F^{C V}\left(c_{1}, c_{2}, \phi\right) & =\lambda_{1} \int_{\Omega}\left|I_{0}(x, y)-c_{1}\right|^{2}(H(\phi(x, y))) d x d y \\
& +\lambda_{2} \int_{\Omega}\left|I_{0}(x, y)-c_{2}\right|^{2}(1-H(\phi(x, y))) d x d y \\
& +\mu \int_{\Omega} \delta(\phi(x, y))|\nabla \phi(x, y)| d x d y,
\end{aligned}
$$

where $\mu \geq 0, \lambda_{1}>0, \lambda_{2}>0$ are fixed constant parameters, $\lambda_{1}$ and $\lambda_{2}$ control the driven force inside and outside the dynamic contour $\phi . \quad \delta(\phi(x, y))=H^{\prime}(\phi(x, y))$ and $c_{1}, c_{2}$ are average intensities of the given image $I_{0}(x, y)$ inside and outside of the evolving curve $\phi$ respectively, and it is given by:

$$
c_{1}(\phi)=\frac{\int_{\Omega} I_{0}(x, y) H(\phi(x, y)) d x d y}{\int_{\Omega} H(\phi(x, y)) d x d y},
$$

if $H(\phi(x, y))>0$ i.e. if the curve has a non empty interior in $\Omega$.

$$
c_{2}(\phi)=\frac{\int_{\Omega} I_{0}(x, y)(1-H(\phi(x, y))) d x d y}{\int_{\Omega}(1-H(\phi(x, y))) d x d y},
$$

if $(1-H(\phi(x, y)))>0$ i.e. if the curve has a nonempty exterior in $\Omega$.

Minimization of (2.1) leads to the following evolution equation

$$
\frac{\partial \phi}{\partial t}=\delta_{\epsilon}(\phi)\left[\mu \nabla \cdot\left(\frac{\nabla \phi}{|\nabla \phi|}\right)-\lambda_{1}\left(I_{0}(x, y)-c_{1}\right)^{2}+\lambda_{2}\left(I_{0}(x, y)-c_{2}\right)^{2}\right] .
$$

Usually regularized Heaviside and dirac delta functions are used and given by:

$$
H_{\epsilon}(\phi)=\frac{1}{2}\left(1+\frac{2}{\pi} \arctan \left(\frac{\phi}{\epsilon}\right)\right), \quad \delta_{\epsilon}(\phi)=\frac{\epsilon}{\pi\left(\epsilon^{2}+\phi^{2}\right)} .
$$

The averages $c_{1}$ and $c_{2}$ (the average prototypes) may disturb and provide different data as compared to original data when an image have inhomogeneity and such a result affects the quality of segmented result. It is important to note that $\mathrm{CV}$ model is not designed for nosy images. Therefore, it may not work properly in noisy images.

2.2. Local Binary Fitting Model (LBF). In order to enhance the result of CV model for images having inhomogeneity, Li et al. [16] proposed local binary fitting (LBF) model by using kernel function and local cluster of pixels instead of using means inside and outside the contour. Let $I_{0}$ be an image defined on $\Omega \subset \Re^{2}$ image domain and $\mathrm{C}$ be the evolving curve and $\phi$ be the contour on image then for any $(x, y)$ the energy functional of the above model is: 


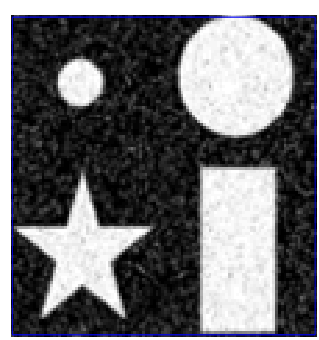

(a) Input Image

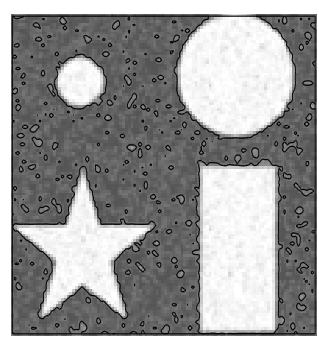

(d) Li Performance

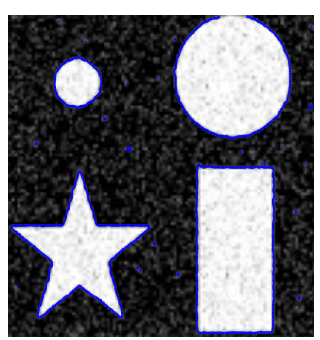

(b) CV performance

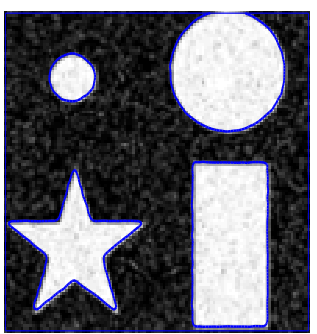

(e) Novel Performance

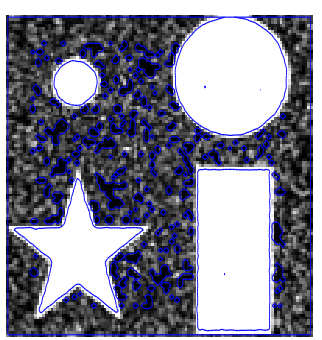

(c) Wu and He Performance

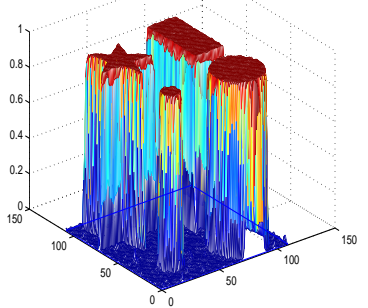

(f) Our 3D plot

Figure 1: The performance comparison of the modernistic model, our model (iteration= 10) $\mathrm{Li}($ iteration $=3000), \mathrm{CV}($ iterations $=150)$ and $\mathrm{Wu}$ and He (iteration= 5000) models on noisy image. .

$$
\begin{aligned}
F^{L B F}\left(\phi, \zeta_{1}, \zeta_{2}\right) & =\lambda_{1} \int_{\Omega} K_{\delta}(x-y) *\left\|I_{0}(x, y)-\zeta_{1}\right\|^{2}(H(\phi(x, y))) d x d y \\
& +\lambda_{2} \int_{\Omega} K_{\delta}(x-y) *\left\|I_{0}(x, y)-\zeta_{2}\right\|^{2}(1-H(\phi(x, y))) d x d y \\
& +\mu \int_{\Omega} \delta(\phi(x, y))|\nabla \phi(x, y)| d x d y
\end{aligned}
$$

where $K_{\delta}(x-y)=\frac{1}{(2 \pi)^{n / 2} \delta^{2}} e^{\frac{-|x-y|^{2}}{2 \delta^{2}}}$, is the Gaussian kernel and $\zeta_{1}, \zeta_{2}$ are local cluster of pixels, computed with the formulas

$$
\zeta_{1}(\phi)=\frac{\int_{\Omega} K_{\delta}(x-y) * H(\phi(x, y)) I_{0}(x, y) d x d y}{\int_{\Omega} K_{\delta}(x-y) * H(\phi(x, y)) d x d y},
$$

and

$$
\zeta_{2}(\phi)=\frac{\int_{\Omega} K_{\delta}(x-y) *(1-H(\phi(x, y))) I_{0}(x, y) d x d y}{\int_{\Omega} K_{\delta}(x-y) *(1-H(\phi(x, y))) d x d y}
$$




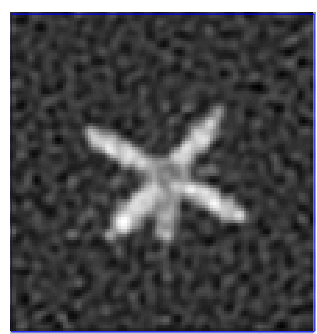

(a) Input Image

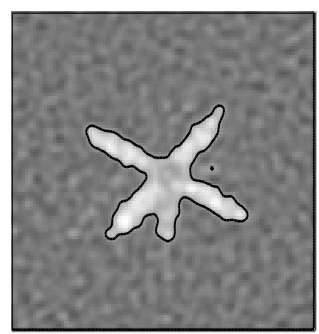

(d) Li Performance

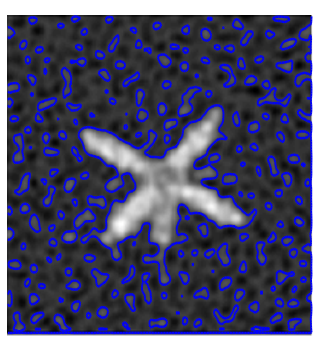

(b) CV Performance

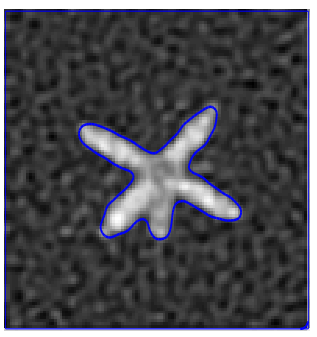

(e) Novel Performance

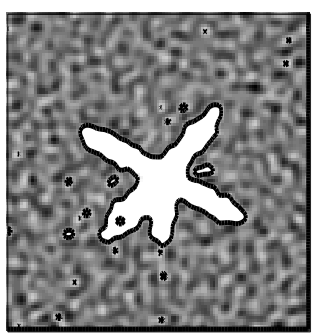

(c) Wu and He Performance

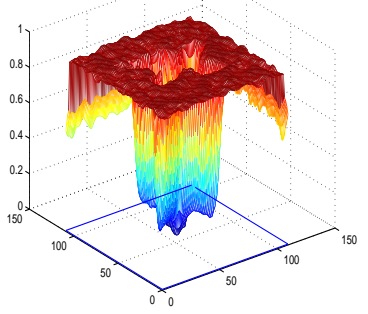

(f) Our 3D plot

Figure 2: The performance comparison of the modernistic model, our model (iteration= 10) $\mathrm{Li}($ iteration $=200), \mathrm{CV}$ (iterations $=200)$ and $\mathrm{Wu}$ and He (iteration= 300) models on noisy image. .

Now keeping $\zeta_{1}$ and $\zeta_{2}$ fixed, and minimizing the energy function with respect to $\phi$ we get;

$$
\frac{\partial \phi}{\partial t}=-\delta_{\varepsilon}(\phi)\left(\lambda_{1} p_{1}-\lambda_{2} p_{2}\right)+\nu \delta_{\varepsilon}(\phi) \operatorname{div}\left(\frac{\nabla \phi}{|\nabla \phi|}\right)+\mu\left(\nabla^{2} \phi-\operatorname{div}\left(\frac{\nabla \phi}{|\nabla \phi|}\right)\right),
$$

where $\delta_{\varepsilon}$ is the smooth Dirac function, and $p_{1}$ and $p_{2}$ are the functions as follow;

$$
\begin{aligned}
& p_{1}=\int_{\Omega} K_{\sigma}(x-y) *\left|I_{0}(x, y)-\zeta_{1}(\phi)\right|^{2} d x d y . \\
& p_{2}=\int_{\Omega} K_{\sigma}(x-y) *\left|I_{0}(x, y)-\zeta_{1}(\phi)\right| d x d y .
\end{aligned}
$$

However this model covers the problem of dealing with an image having intensity inhomogeneity but as this model utilizing the local information which make this model unable in working with those images having noise. This model is also not able to segment the image having high contrast of inhomogeneity. The limitation of this model can be seen in those images where there is multi-intensities objects having average intensity background, furthermore this model is not capable of capturing required target object. 


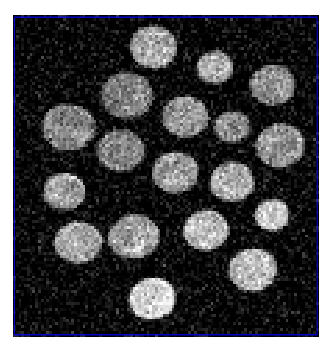

(a) Input Image

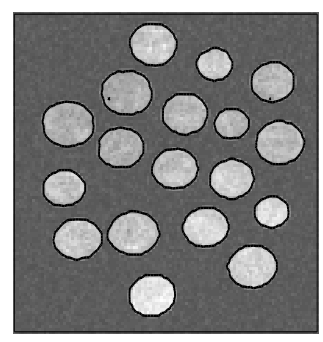

(d) Li Performance

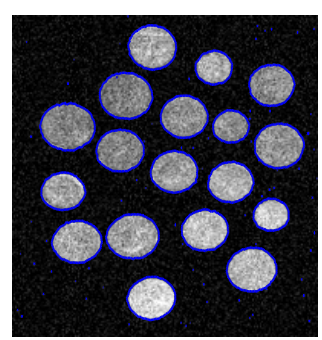

(b) CV Performance

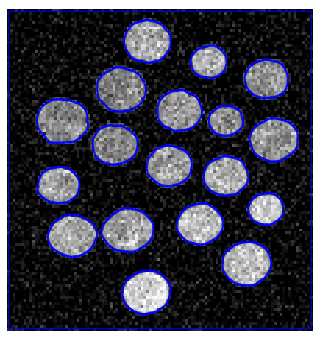

(e) Novel Performance

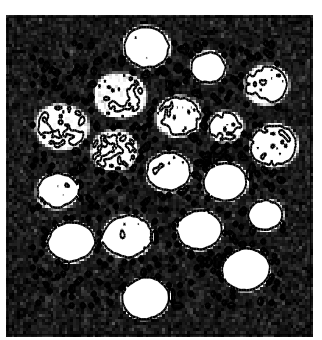

(c) Wu and He Performance

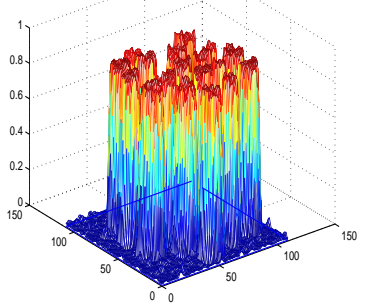

(f) Our 3D plot

Figure 3: The performance comparison of the modernistic model, our model (iteration= 10) $\mathrm{Li}($ iteration $=200), \mathrm{CV}$ (iterations $=150)$ and $\mathrm{Wu}$ and He (iteration= 300) models on additive noise (gaussian 0.001). .

2.3. Convex Variational Level Set Model. Wu and He [25] proposed a convex model based on the coefficient of variation, whose energy functional is:

$$
\begin{aligned}
F^{W U}(\phi) & =\lambda \int_{\Omega} \frac{\left(I_{0}(x, y)-c_{1}\right)^{2}}{c_{1}^{2}}(1+\phi)^{2} d x d y \\
& +\int_{\Omega} \frac{\left(I_{0}(x, y)-c_{2}\right)^{2}}{c_{2}^{2}}(1-\phi)^{2} d x d y
\end{aligned}
$$

where $c_{1}$ and $c_{2}$ are average intensities inside and outside of the evolving curve $\phi$ respectively in the given image $I_{0}(x, y)$. This model guarantee for any image the value of unique global minimizer for the energy functional lies in [-1, 1], and for any binary ideal image it is equal to -1 in the background and 1 in the object. However this model is convex so it is independent of initial contour but this model may not work properly in noisy images. This model fails to isolate the desired object from the given image.

2.4. Geodesic Active Contour Model. C. L. Guyader and C. Gout.[15] proposed an model which is based on the geometric constraint. The aim of this model 


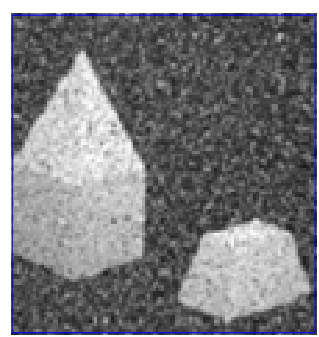

(a) Input Image

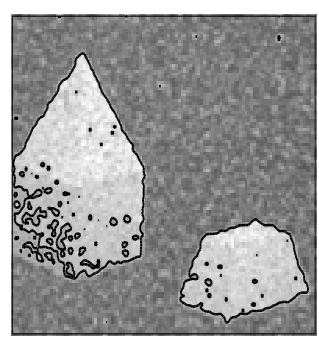

(d) Li Performance

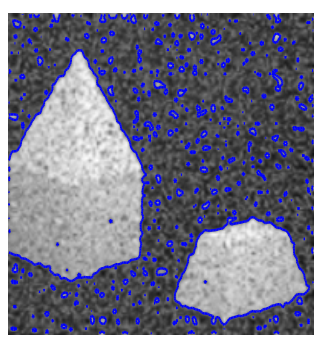

(b) CV Performance

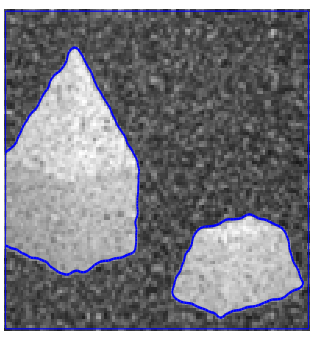

(e) novel Performance

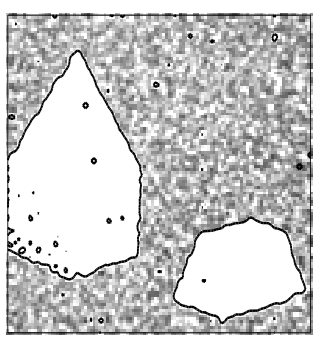

(c) Wu and He Performance

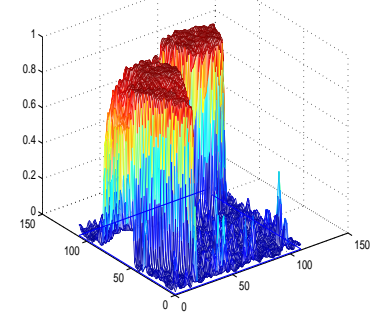

(f) Our 3D plot

Figure 4: The performance comparison of the modernistic model, our model (iteration= 10) $\mathrm{Li}($ iteration $=200), \mathrm{CV}$ (iterations $=130)$ and $\mathrm{Wu}$ and He (iteration= 300) models on noisy image. .

is to extract the desired object from the given image, which is mathematically given as:

$$
A=\left\{\left(x_{i}, y_{i}\right) \epsilon \Omega, 1 \leq i \leq n\right\} \subset \Omega .
$$

To find the contour that has best approach towards the points $A$, this model uses the edge detector function $g$ and distance function $d$. Normally the following choices are used:

$$
g\left(\left|\nabla I_{0}\right|\right)=\frac{1}{1+\left|\nabla I_{0}\right|^{2}} .
$$

and,

$$
d(x, y)=\prod_{i=1}^{3}\left(1-e^{\frac{-\left[(x-x i)^{2}+(y-y i)^{2}\right]}{2(\sigma)^{2}}}\right) \forall(x, y) \epsilon \Omega
$$




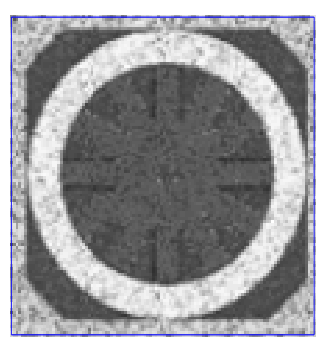

(a) Input Image

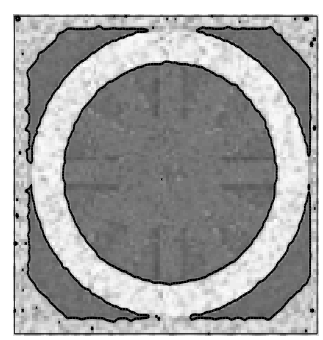

(d) Li Performance

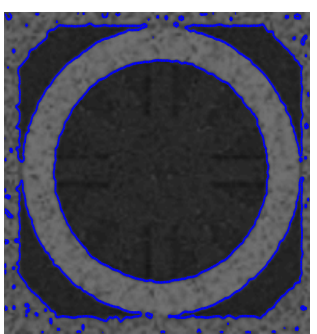

(b) CV Performance

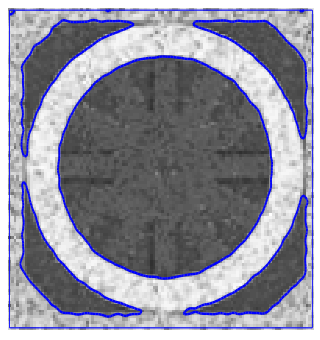

(e) Novel Performance

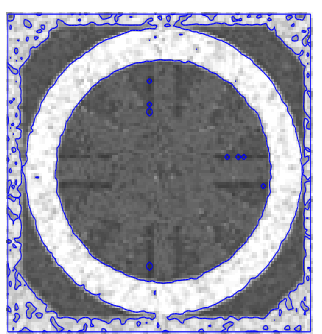

(c) Wu and He Performance

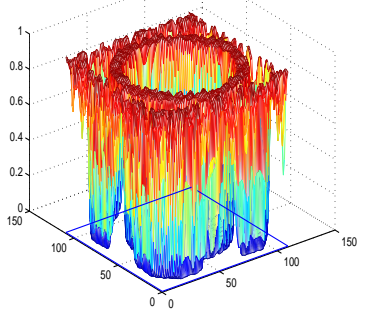

(f) Our 3D plot

Figure 5: The performance comparison of the modernistic model, our model (iteration= 10) $\mathrm{Li}($ iteration $=200), \mathrm{CV}$ (iterations $=150)$ and $\mathrm{Wu}$ and He (iteration= 300) models on noisy image. .

one can observe that $d \approx 0$ near the points chosen and $g \approx 0$ on all the edges. In order to stop the evolving $\phi$ on boundary of the desired object i.e. where $g \approx 0$ and $d \approx 0$, this model needs the minimization of the following energy functional.

$$
F^{\text {Gout }}(\phi)=\int_{\Omega} d(x, y) g\left(\left\|\nabla I_{0}(x, y)\right\|\right) d x d y
$$

By applying level set function on the above energy functional the domain of an image will be extended to image domain $\Omega$.

In terms of level set formulation the above energy functional is represented as:

$$
F^{\text {Gout }}(\phi)=\int_{\Omega} d(x, y) g\left(\left\|\nabla I_{0}(x, y)\right\|\right)\|\nabla H(\phi(x, y))\| d x d y
$$

where length of the contour $\phi$ is represented by:

$$
\int_{\Omega}\|\nabla H(\phi(x, y))\| d x d y
$$




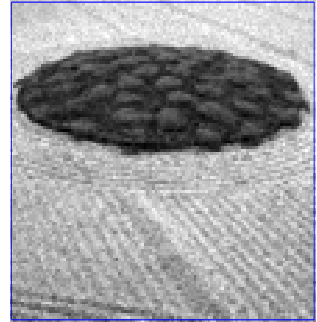

(a) Input Image

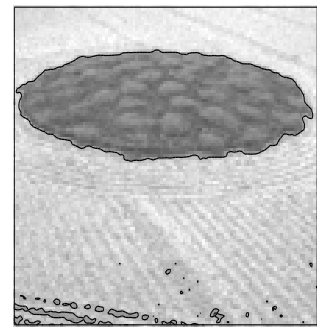

(d) Li Performance

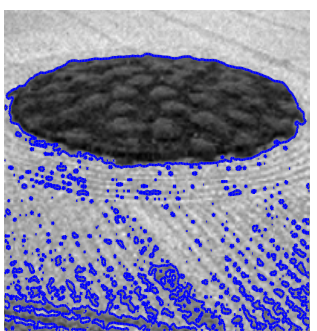

(b) CV Performance

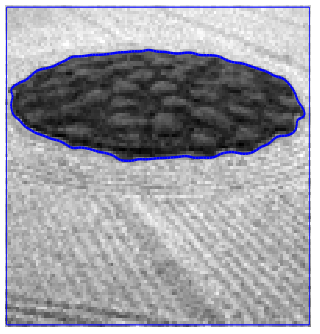

(e) Our Linear Performance

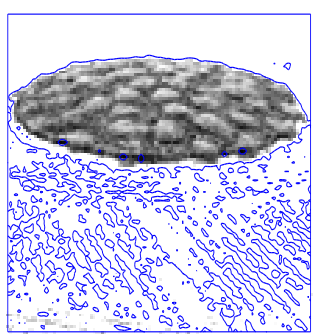

(c) Wu and He Performance

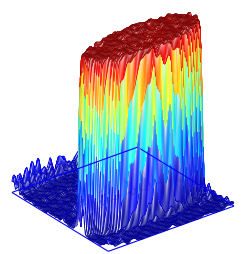

(f) Our 3D plot

Figure 6: The performance comparison of the modernistic model, our model (iteration= 10) $\mathrm{Li}($ iteration $=200), \mathrm{CV}$ (iterations $=150)$ and $\mathrm{Wu}$ and He (iteration= 300) models on additive noise (speckle 0.001) .

The minimization of $(2.17)$ with respect to $\phi$ leads to the following equation:

$$
-\delta_{\epsilon}\left(\phi(x, y) d i v\left[d(x, y) g\left(\left\|\nabla I_{0}(x, y)\right\|\right)\left(\frac{\nabla \phi(x, y)}{|\nabla \phi(x, y)|}\right)\right]=0 .\right.
$$

Thus the evolution equation of this model is:

$$
\frac{\partial \phi(x, y, t)}{\partial t}=\delta_{\Omega}(\phi(x, y, t)) d i v\left[d(x, y) g\left(\left\|\nabla I_{0}(x, y)\right\|\right)\left(\frac{\nabla \phi(x, y, t)}{|\nabla \phi|}\right)\right],
$$

with the boundary condition given as:

$$
\frac{\delta_{\epsilon}(\phi(x, y, t))}{|\nabla \phi(x, y, t)|} \frac{\partial \phi(x, y, t)}{\partial \vec{n}}=0 .
$$

Because Heaviside function is not differentiable, regularized Heaviside and dirac delta functions are used.

\section{The Proposed Model}

The models discussed in the background are not able to perform task of image segmentation for noisy images or local image segmentation. For this purpose we 


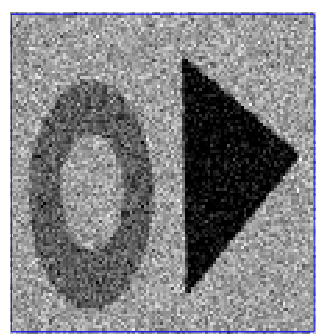

(a) Input Image

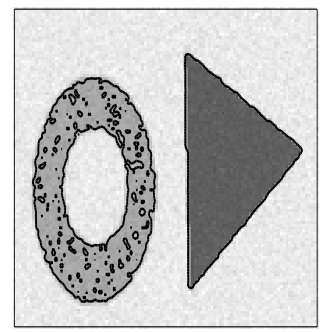

(d) Li Result

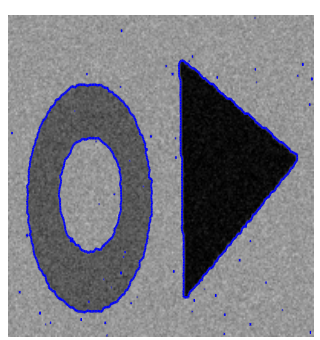

(b) CV Result

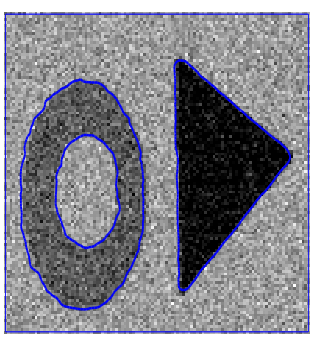

(e) Our Linear Result

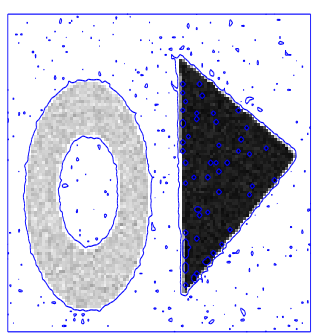

(c) Wu and He Result

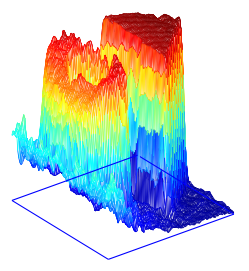

(f) Our 3D plot

Figure 7: The performance comparison of the modernistic model, our model (iteration= 10) $\mathrm{Li}($ iteration $=200), \mathrm{CV}$ (iterations $=100)$ and $\mathrm{Wu}$ and He (iteration= 300) models on additive noise (gaussian 0.001) .

propose modernistic model which work on noisy images and also able to perform task of capturing particular object in the given image.

3.1. Global Segmentation And De-noising Model. In this stage we propose a modernistic variational segmentation model [7] for segmenting image having high noise. The main objective of the proposed model is to handle the image having unwanted signals (noise) and segment simultaneously to get a clean segmented result. Now, encouraged from the work done in background, we are in state to describe our new proposed novel variational model $[19,4,8,1]$ based on kernel function in the framework of active contour, for segmenting noisy images. We use convolution which helps in adding each element of image into local neighborhood of the kernel function. If images have multiple objects with different variation in intensities i.e. maximum, minimum or average intensity back-ground this model works. Let image $I_{0}$ be defined on $\Omega \subset \Re^{2}$ and $\phi$ be the evolving curve then our energy functional is: 


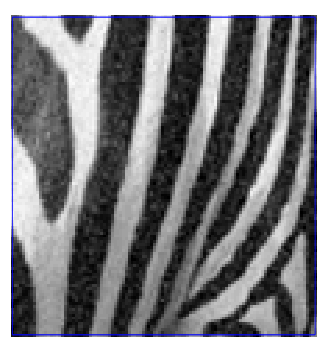

(a) Input Image

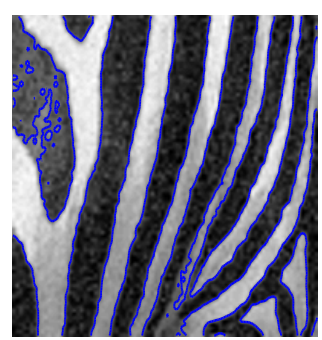

(b) CV Performance

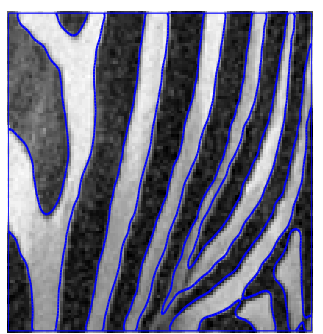

(e) Our Linear Performance

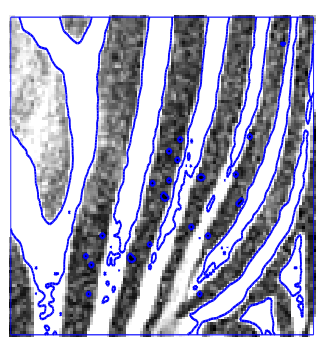

(c) Wu and He Performance

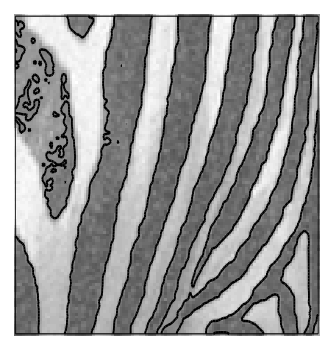

(d) Li Performance

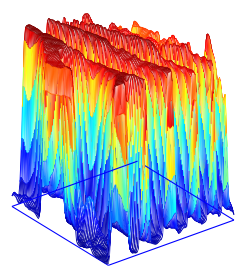

(f) Our 3D plot

Figure 8: The performance comparison of the modernistic model, our model (iteration= $10), \mathrm{Li}($ iteration $=200), \mathrm{CV}$ (iterations $=150)$ and $\mathrm{Wu}$ and He (iteration= 300) models on noisy image .

$$
\begin{array}{r}
F\left(\phi, c_{1}, c_{2}\right)=\int_{\Omega} \int_{\Omega} K_{\delta}(x-y)\left(I_{0}(x, y)-c_{1}\right)^{2} \phi^{2}(x) d x d y+ \\
\int_{\Omega} \int_{\Omega} K_{\delta}(x-y)\left(I_{0}(x, y)-c_{2}\right)^{2}(1-\phi(x))^{2} d x d y
\end{array}
$$

where Gaussian kernel function for $\delta>0$ is defined as:

$$
K_{\delta}(x-y)=\frac{1}{(2 \pi)^{n / 2} \delta^{2}} e^{\frac{-|x-y|^{2}}{2 \delta^{2}}} .
$$

$\phi$ can be calculated by keeping $c_{1}$ and $c_{2}$ constant as follow:

$$
\phi=\frac{K_{\delta}(x-y) *\left(I_{0}(x, y)-c_{2}\right)}{K_{\delta}(x-y) *\left(\left(I_{0}(x, y)-c_{1}\right)+\left(I_{0}\left((x, y)-c_{2}\right)\right)\right)},
$$

$c_{1}$ and $c_{2}$ can be computed as follow:

$$
c_{1}=\frac{\int_{\Omega} I_{0}(x, y) K_{\delta}(x-y) * \phi^{2}(x)}{\int_{\Omega} K_{\delta}(x-y) * \phi^{2}(x)},
$$




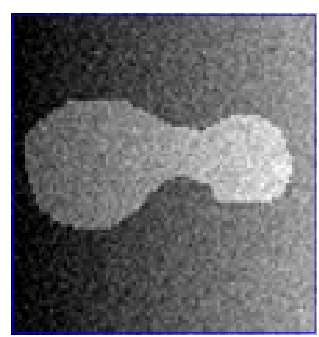

(a) Input Image

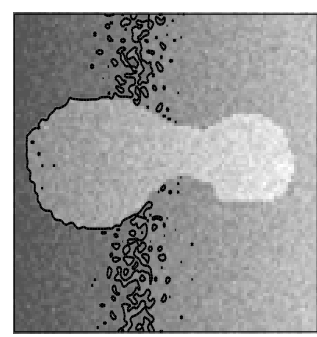

(d) Li Performance

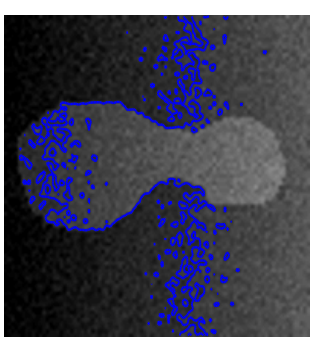

(b) CV Performance

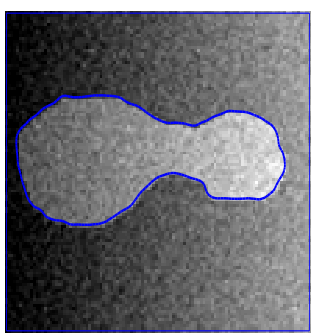

(e) Our Linear Performance

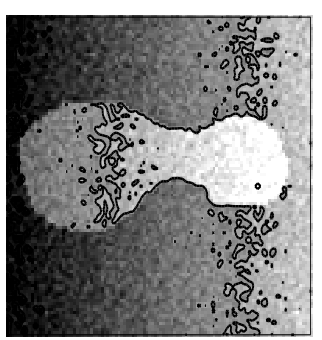

(c) Wu and He Performance

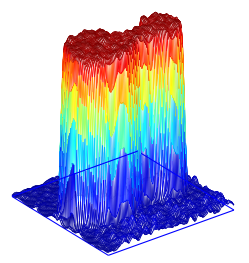

(f) Our 3D plot

Figure 9: The performance comparison of the modernistic model, our model (iteration= $10), \mathrm{Li}($ iteration $=200), \mathrm{CV}$ (iterations $=150)$ and $\mathrm{Wu}$ and $\mathrm{He}($ iteration $=300)$ models on noisy image. .

$$
c_{2}=\frac{\int_{\Omega} I_{0}(x, y) K_{\delta}(x-y) *(1-\phi(x))^{2}}{\int_{\Omega} K_{\delta}(x-y) *(1-\phi(x))^{2}},
$$

under the influence of noise the proposed model alleviates the negative effect of outlier and in the result it gives clear segmented image.

3.2. Piecewise Linear Approach. In noisy image our model with linear approach give nice and clean result for segmentation.

$$
\begin{array}{r}
F^{\text {linear }}(\phi)=\int_{\Omega} \int_{\Omega} K_{\delta}(x-y)\left(I_{0}(x, y)-\ell_{1}\right)^{2} \phi^{2}(x) d x d y \\
+\int_{\Omega} \int_{\Omega} K_{\delta}(x-y)\left(I_{0}(x, y)-\ell_{2}\right)^{2}(1-\phi(x))^{2} d x d y
\end{array}
$$




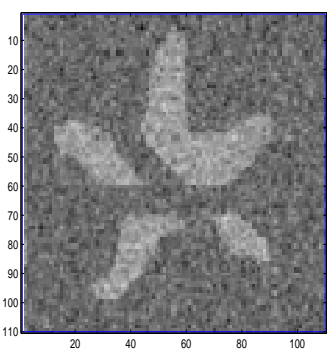

(a) Input Image

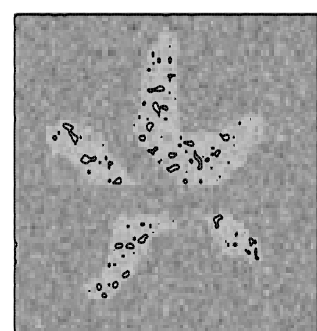

(d) Li Result

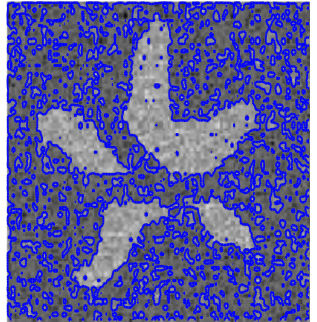

(b) CV Performance

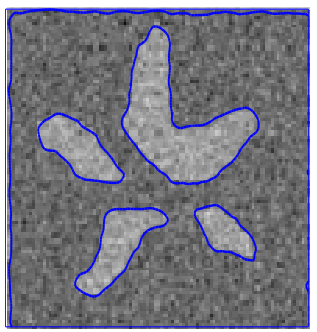

(e) Our Linear Performance

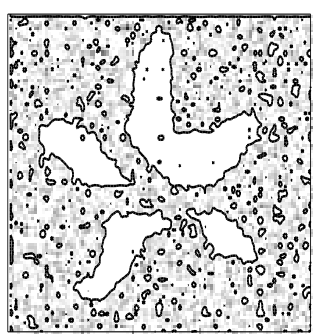

(c) Wu and He Performance

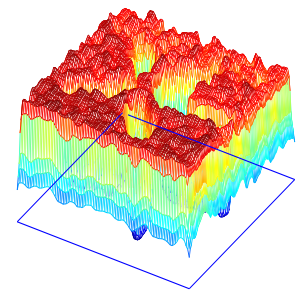

(f) Our 3D plot

Figure 10: The performance comparison of the modernistic model, our model (iteration= $10), \mathrm{Li}($ iteration $=200), \mathrm{CV}$ (iterations $=150)$ and $\mathrm{Wu}$ and $\mathrm{He}($ iteration $=300)$ models on noisy image. .

$$
\begin{array}{r}
F^{\text {linear }}\left(\phi, c, c_{1}, c_{2}, p_{0}, p_{1}, p_{2}\right)=\int_{\Omega} \int_{\Omega} K_{\delta}(x-y)\left(I_{0}(x, y)-c-c_{1} x-c_{2} y\right)^{2} \phi^{2}(x) d x d y \\
+\int_{\Omega} \int_{\Omega} K_{\delta}(x-y)\left(I_{0}(x, y)-p_{0}-p_{1} x-p_{2} y\right)^{2}(1-\phi(x))^{2} d x d y .
\end{array}
$$

Here, $l_{1}=c+c_{1 x}+c_{2 y}$ and $l_{2}=P_{0}+P_{1 x}+P_{2 y}$ are basically linear functions they work better than the constant function when their is noise and when their is intensity inhomogeneity. By keeping all the coefficients of a linear function constant $\phi$ can be calculated as follow:

$$
\phi=\frac{K_{\delta}(x-y) *\left(I_{0}(x, y)-l_{2}\right)^{2}}{K_{\delta}(x-y) *\left[\left(I_{0}(x, y)-l_{1}\right)^{2}+\left(I_{0}(x, y)-l_{2}\right)^{2}\right]} .
$$

All the $c_{i} s$ for $i=\{0,1,2\}$ can be calculated by linear system:

$$
\frac{\partial}{\partial c_{i}}\left[K_{\delta}(x, y)\left(I_{0}(x, y)-c-c_{1} x-c_{2} y\right)^{2} \phi^{2}(x)\right]=0 .
$$


Similarly all the $p_{i} s$ for $i=\{0,1,2\}$ can be calculated by linear system:

$$
\frac{\partial}{\partial p_{i}}\left[K_{\delta}(x-y)\left(I_{0}(x, y)-p_{0}-p_{1} x-p_{2} y\right)^{2}(1-\phi(x))^{2}\right]=0 .
$$

3.3. Quadratic Approach. Further, we present algorithm for segmenting the images in the presence of noise by using quadratic gray-level function.

$$
\begin{gathered}
F\left(\phi, c, c_{1}, c_{2}, c_{3}, c_{4}, c_{5}, p_{0}, p_{1}, p_{2}, p_{3}, p_{4}, p_{5}\right)= \\
\int_{\Omega} \int_{\Omega} K_{\delta}(x-y)\left(I_{0}(x, y)-\ell_{1}\right)^{2} \phi^{2}(x) d x d y+ \\
\int_{\Omega} \int_{\Omega} K_{\delta}(x-y)\left(I_{0}(x, y)-\ell_{2}\right)^{2}(1-\phi(x))^{2} d x d y,
\end{gathered}
$$

$F\left(\phi, c, c_{1}, c_{2}, c_{3}, c_{4}, c_{5}, p_{0}, p_{1}, p_{2}, p_{3}, p_{4}, p_{5}\right)(3.33)$

$$
\begin{gathered}
\int_{\Omega} \int_{\Omega} K_{\delta}(x-y)\left(I_{0}(x, y)-c_{5} x^{2}-c_{4} y^{2}-c_{3} x y-c_{2} x-c_{1} y-c\right)^{2} \phi^{2}(x) d x d y+(3.34) \\
\int_{\Omega} \int_{\Omega} K_{\delta}(x-y)\left(I_{0}(x, y)-p_{5} x^{2}-p_{4} y^{2}-p_{3} x y-p_{2} x-p_{1} y-p_{0}\right)^{2}(1-\phi(x))^{2} d x d y .
\end{gathered}
$$

By keeping all the coefficients of quadratic function constant $\phi$ is computed as:

$$
\phi=\frac{K_{\delta}(x-y) *\left(I_{0}(x, y)-l_{2}\right)^{2}}{K_{\delta}(x-y) *\left[\left(I_{0}(x, y)-l_{1}\right)^{2}+\left(I_{0}(x, y)-l_{2}\right)^{2}\right]},
$$

where

$$
l_{1}=c_{5} x^{2}+c_{4} y^{2}+c_{3} x y+c_{2} x+c_{1} y+c .
$$

and

$$
l_{2}=p_{5} x^{2}+p_{4} y^{2}+p_{3} x y+p_{2} x+p_{1} y+p_{0} .
$$

Clearly $l_{1}$ and $l_{2}$ are quadratic functions and they much better than the constant function $c_{1}$ and $c_{2}$ when their is noise and intensity inhomogeneity or both at the same time, so we use $l_{1}$ and $l_{2}$ and these are also options for us.

All the $c_{i} s$ for $i=\{0,1,2,3,4,5\}$ can be calculated by the system:

$$
\frac{\partial}{\partial c_{i}}\left[K_{\delta}(x, y)\left(I_{0}(x, y)-c_{5} x^{2}-c_{4} y^{2}-c_{3} x y-c_{2} x-c_{1} y-c\right)^{2} \phi^{2}(x)\right]=0 .
$$

Similarly all the $p_{i} s$ for $i=\{0,1,2,3,4,5\}$ can be calculated by the system:

$$
\frac{\partial}{\partial p_{i}}\left[K_{\delta}(x-y)\left(I_{0}(x, y)-p_{5} x^{2}-p_{4} y^{2}-p_{3} x y-p_{2} x-p_{1} y-p_{0}\right)^{2}(1-\phi(x))^{2}\right]=0 .
$$


3.4. Selective Segmentation Approach. The problem arises in image segmentation is to segment only the desire object among the all objects in image having same intensities. Many models have been proposed for selective segmentation[23, 2], but in case of noise there performance is not satisfactory. Now to extend our work to segment the desire objects in presence of noise we use weight function as in the model [10]. let $I_{0}(x, y)$ be the given image defined on a rectangular domain $\Omega$. Consider the set of $n_{1}$ points near the object's boundary we want to detect, which is given as:

$$
A=\left\{\left(x_{i}, y_{i}\right) \epsilon \Omega, 1 \leq i \leq n\right\} \subset \Omega .
$$

To detect the desire object in image we are required to find a contour that approaches best towards the point from the set A. To detect the selective object we use weight function which is $w(x, y)=1-d(x, y) g$, and the distance function $d(x, y)$ which is defined as:

$$
d(x, y)=\prod_{i=1}^{3}\left(1-e^{\frac{-\left[(x-x i)^{2}+(b-y i)^{2}\right]}{2(\sigma)^{2}}}\right)
$$

It represents geometric constraints and restrict the evolving curve to move away from the points of set A. The well known edge detector function is use to detect the edges of the object is:

$$
g\left(\left|\nabla I_{0}\right|\right)=\frac{1}{1+\left|\nabla I_{0}\right|^{2}}
$$

that is:

$$
g\left(\left|\nabla I_{0}\right|\right)=\frac{1}{1+\left(I_{0 x}^{2}+I_{0 y}^{2}\right)} .
$$

For local segmentation in presence of noise our energy functional will be:

$$
\begin{array}{r}
F\left(\phi, c_{1}, c_{2}\right)=\int_{\Omega} \int_{\Omega} K_{\delta}(x-y) w(x, y)\left(I_{0}(x, y)-c_{1}\right)^{2} \phi^{2}(x) d x d x+ \\
\int_{\Omega} \int_{\Omega} K_{\delta}(x-y) w(x, y)\left(I_{0}(x, y)-c_{2}\right)^{2}(1-\phi(x))^{2} d x d y
\end{array}
$$

$\phi$ can be calculated by keeping $c_{1}$ and $c_{2}$ constant as follow:

$$
\phi=\frac{K_{\delta}(x-y) * w(x, y)\left(I_{0}(x, y)-c_{2}\right)}{K_{\delta}(x-y) * w(x, y)\left(\left(I_{0}(x, y)-c_{1}\right)+\left(I_{0}(x, y)-c_{2}\right)\right)} .
$$

For $c_{1}$ and $c_{2}$ we have:

$$
c_{1}=\frac{I_{0} * w(x, y) * K_{\delta}(x-y) * \phi^{2}(x)}{w(x, y) * K_{\delta}(x-y) * \phi^{2}(x)} .
$$


Table 1: Jaccard Similarity comparison table of the Our Method, Wu-He Method, Li Method and CV Method.

\begin{tabular}{||c|c|c|c|c|c|c||c|c||}
\hline \multirow{2}{*}{ Image Size } & \multicolumn{2}{|c|}{ Our Method } & \multicolumn{2}{c|}{ Wu-He Method } & \multicolumn{2}{c|}{ Li Method } & \multicolumn{2}{c||}{ CV Method } \\
\cline { 2 - 9 } & Fig & Js & Fig & Js & Fig & Js & Fig & Js \\
\hline $250 \times 250$ & 1 & 0.9977 & 1 & 0.5866 & 1 & 0.6023 & 1 & 0.8023 \\
\hline $250 \times 250$ & 2 & 0.9087 & 2 & 0.4536 & 2 & 0.7907 & 2 & 0.4907 \\
\hline $250 \times 250$ & 3 & 0.987 & 3 & 0.6463 & 3 & 0.8532 & 3 & 0.9532 \\
\hline $250 \times 250$ & 4 & 0.9281 & 4 & 0.4389 & 4 & 0.3695 & 4 & 0.5695 \\
\hline $250 \times 250$ & 5 & 0.9761 & 5 & 0.6544 & 5 & 0.7870 & 5 & 0.8870 \\
\hline $250 \times 250$ & 6 & 0.8633 & 3 & 0.5073 & 6 & 0.7733 & 6 & 0.6733 \\
\hline $250 \times 250$ & 7 & 0.9376 & 4 & 0.5735 & 7 & 0.7553 & 7 & 0.8553 \\
\hline $250 \times 250$ & 8 & 0.95370 & 5 & 0.5571 & 8 & 0.7241 & 8 & 0.6241 \\
\hline
\end{tabular}

$$
c_{2}=\frac{I_{0} * w(x, y) * K_{\delta}(x-y) *(1-\phi(x))^{2}}{w(x, y) * K_{\delta}(x-y) *(1-\phi(x))^{2}} .
$$

The principle stride of our model for quadratic approach is as follows:

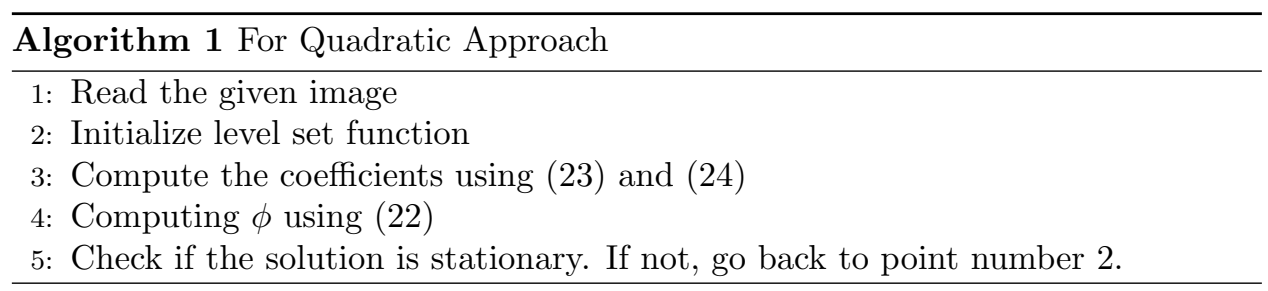




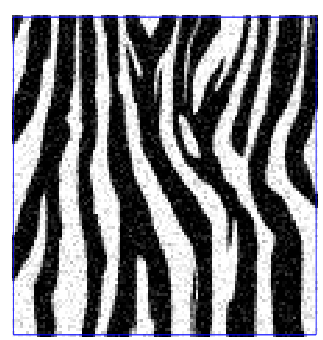

(a) Input Image

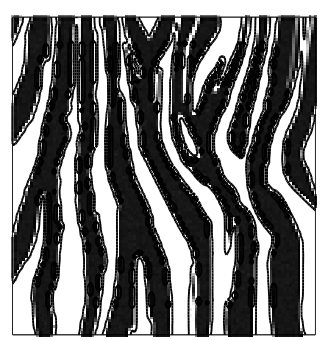

(b) Wu and He Performance

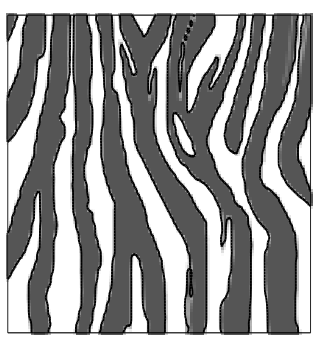

(c) Li Performance

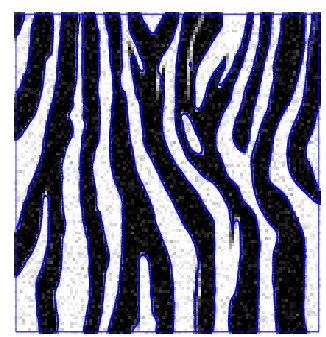

(d) Our Quadratic Performance

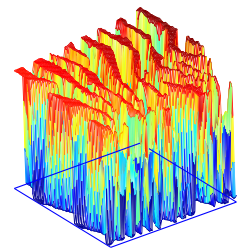

(e) Our 3D plot

Figure 11: The performance comparison of the modernistic model, our model (iteration= $50), \mathrm{Li}($ iteration $=200)$ and $\mathrm{Wu}$ and He (iteration= 300) models on noisy image. .

\section{Experimental Results}

In this section we give some experimental results on different data set in order to explain that our model is new and performs well as compared to other existing models in presence of outlier. In CV model [6] with length term works to segment object but unable to segment those images having noise. Our model performs the task of segmentation [26] in both presence and absence of noise. Therefore, if we compare our model with the other models it performs well and give a good experimental results. To obtain result of our model we used MATLAB installed in personal computer with specification: windows 8.1, 4.00GB installed memory (RAM) and $1.80 \mathrm{GHz}$ processor.

Test set 1: In first test set we show the performance of our model in the segmentation of gray image having noise. These test supports in comparison to Chan-Vese model with fitting term in gray-scale image. Further we will do comparison with $\mathbf{W u}$ and He model and with LBF model [13, 25] which shows better performance. Our technique has been applied to naturally out door images. Few example are display in Fig. 1, 2, 3, 4, and 5 


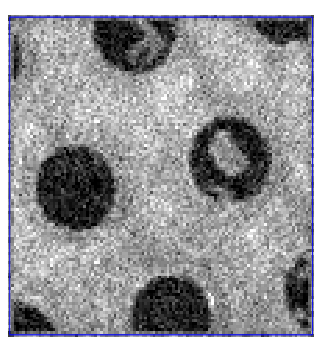

(a) Input Image

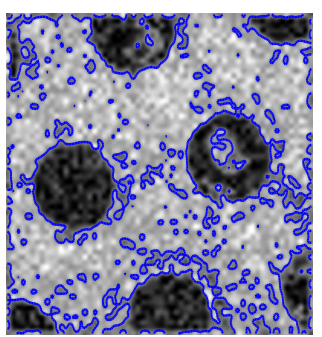

(b) CV Performance

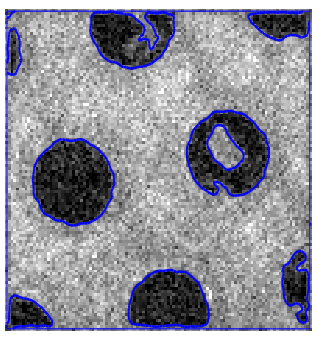

(e) Our Quadratic Performance

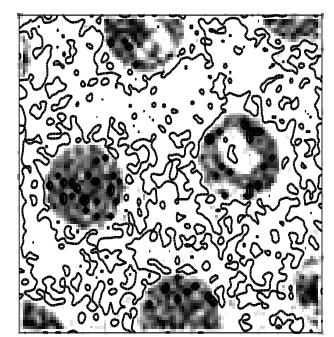

(c) Wu and He Performance

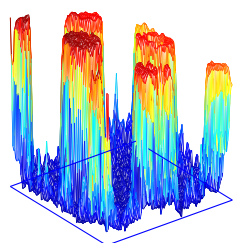

(f) Our 3D plot

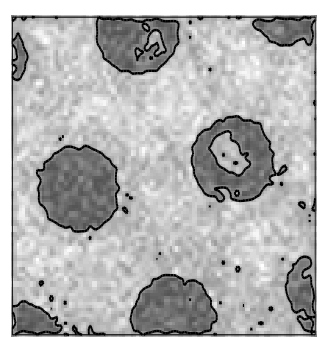

(d) Li Performance

Figure 12: The performance comparison of the modernistic model, our model (iteration= $100), \mathrm{Li}($ iteration $=200), \mathrm{CV}$ (iterations $=150)$ and $\mathrm{Wu}$ and He (iteration= 300) models on gaussian noise 0.001 .

Test set 2: In second test set we show the performance of our model with piecewise linear approach on noisy image, also we add some noise such as gaussian noise and salt and pepper on clear image to disturb the image signals and justify the model. First we compare it with our global model and then with CV Model, $\mathrm{Wu}$ and $\mathrm{He}$ model and $\mathrm{Li}$ et al model. Also we conclude that our model in piecewise linear approach work in some inhomogeneity and give best performance, Few examples are display in Fig 6,7,8,9 and 10.

Test set 3: In third test we show the performance of our model with quadratic approach in presence of noise, the test result are display in fig 11 and 12 . The comparison of our all approaches is shown in Fig 13. Further in local image segmentation case, Some test show that our model also work in selective segmentation [3] of noisy images simultaneously, the result is display in Fig 14.

\section{Conclusions}

In this article different from the other variational models, we proposed the model to: 


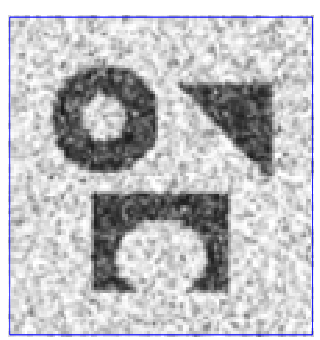

(a) Input Image

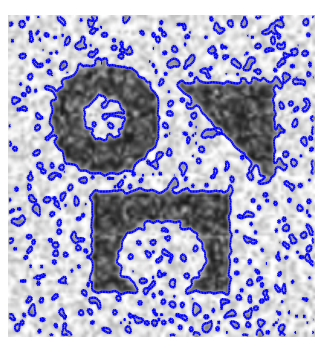

(b) CV Performance

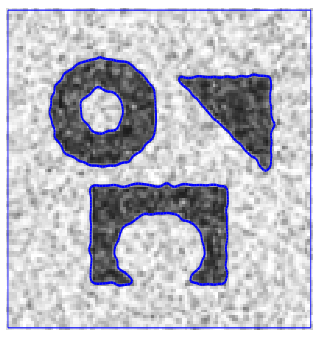

(e) novel Performance

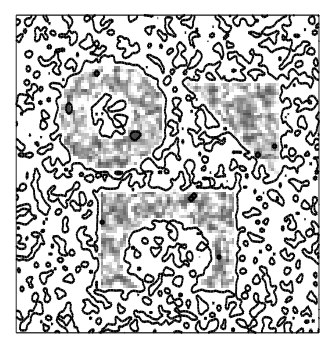

(c) Wu and He Performance

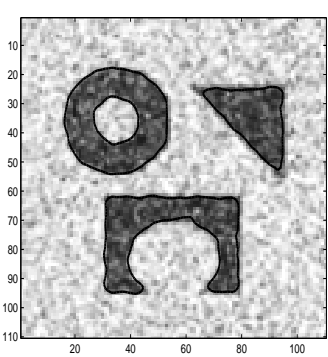

(f) Our Quadratic Performance

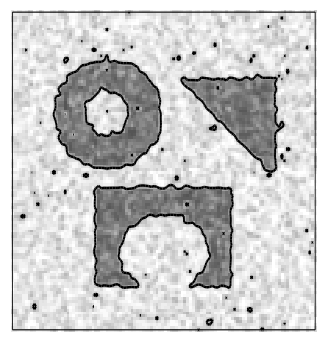

(d) Li Performance

Figure 13: The performance comparison of the modernistic model, our model (iteration= 10) $\mathrm{Li}($ iteration $=200), \mathrm{CV}$ (iterations $=170)$ and $\mathrm{Wu}$ and He (iteration= 300) models on noisy image. .

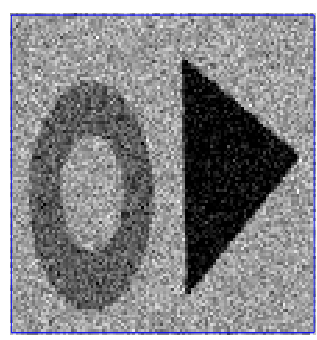

(a) Input Image

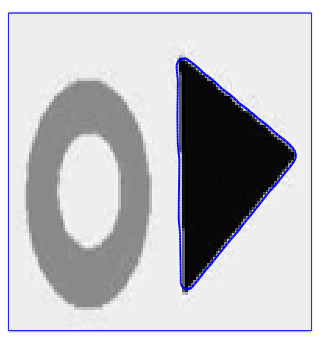

(b) Our selective Performance

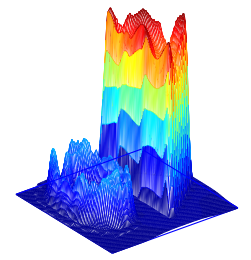

(c) Our 3D plot

Figure 14: Given figure demonstrate the performance of the proposed model on selective segmentation. . 
(1) Diminish the negative effects of outlier.

(2) Perform selective segmentation.

(3) Segment noise images.

(4) Segment image with intensity inhomogeneity.

(5) Segment image with intensity inhomogeneity and noise together.

Using the characteristic of kernel function for image segmentation, further weight function is used in the proposed model for local segmentation of noisy images. In order to enhance the result of our model we use different approaches in the proposed model i.e. piecewise linear approach and then quadratic approach. Our model is based on level set formulation $[20,10]$ in which the edge of the objects is the zero level set of $\phi$. The minimization of our modernistic model with respect to $\phi$ is derive by Euler Lagrange's equation [21]. The functions $c_{1}$ and $c_{2}$ are calculated first and then $\phi$ is computed analytically. The values of $c_{1}$ and $c_{2}$ are also calculated by Euler Lagrange's equation. To run the entire process analytically on images the software we have used is MATLAB. We test our model on different outdoor and medical images and we have observe its performance. Further we compare the performance of our novel model with state of the art models. We conclude from different experiment results that our model is performing better than Chan-Vese model [6], Li et al. model [13] and Wu and He [25] model qualitatively.

\section{REFERENCES}

[1] G. Aubert and L. Vese A Variational Method in Image Recovery, SIAM Journal on Numerical Analysis, 34 (1997) 1948-1979.

[2] G. Aubert and P.Kornprobst, Mathematical problems in image processing: Partial Differential Equations and the Calculus of Variations, Springer, 2002.

[3] N. Badshah and K. Chen, H. Ali and G. Murtaza Coefficient of Variation Based Image Selective Segmentation Using Active Contour, East Asian Journal on Applied Mathematics, 2, No. 2 (2012) 150-169.

[4] N. Badshah and K. Chen Image Selective Segmentation under Geometrical Constraints Using an Active Contour Approach, Mathematics of Computations, 7 (2010) 759-778.

[5] N. Badshah, Fast iterative methods for variational models in image segmentation. Ph.D. Thesis, Liverpool University, Liverpool, England, 2009.

[6] J. Chen and T. N. Pappas and A. Mojsilovic and B. E. Rogowitz Adaptive Perceptual Color Texture Image Segmentation, IEEE Transactions on Image Processing, 14 (2005)1524-1536.

[7] T. F. Chan and L. A. Vese Active Contour Without Edges, IEEE Transactions on Image Processing, 10,(2001) 266-277.

[8] T. C. Chan and J. J. Shen and L. Vese Variational PDE Models in Image Processing, Notices of the American Mathematical Society, 50 (2003) 14-26.

[9] T. F. Chan and Y. B. Sandberg and V. A. Luminita Active Contours Without Edges for Vector-Valued Images, Journal of Visual Communication and Image Representation, 11, No. 2 (2000) 130-141.

[10] S. Christoph A Study of a Convex Variational Diffusion Approach for Image Segmentation and Feature Extraction, Journal of Mathematical Imaging and Vision, 8(1998) 271-292.

[11] N. Dhanachandra and K. Manglem and Y. J. Chanu Image Segmentation using K-means Clustering Algorithm and Subtractive Clustering Algorithm, Procedia Computer Science, 54 (2015) 764-771.

[12] C. Gout and C. L. Guyader and L. A. Vese Segmentation under Geometrical Conditions with Geodesic Active Contour and Interpolation using Level Set Method, Numerical Algorithms, 39 (2005) 155-173. 
[13] C. L. Guyader and C. Gout Geodesic Active Contour under Geometrical Condition: Theory and 3D Appliction, Numerical Algorithms, 48 (2008) 105-133.

[14] A. Jose and S. Ravi and M. Sambath Brain Tumor Segmentation using K-means Clustering and Fuzzy C-means Algorithm and its Area Calculation, Interational Journal of Innovative Research in Computer and Communication Engineering, 2, No. 2 (2014) 60-64.

[15] M. S. Khan and H. Ali, N. Badshah and G. Ayub Khan Convex Active Contours with Locally Bias Field Estimator, Punjab Uni. j.math. 52, No. 10 (2020) 81-114.

[16] M. Kass and A. Witkin and D. Terzopouls Active Contours Models, International Journal of Computer Vision, 1 (1988) 321-331.

[17] C. Li and C. Y. Kao and J. C. Gore and Z. Ding Minimization of Region Scalable Fitting Energy for Image Segmentation, IEEE Transactions on Image Processing, 17 (2008) 1940-1949.

[18] C. Li and C. Y. Kao and J. C. Gore and Z. Ding Implicit Active Contours Driven by Local Binary Fitting Energy, In proceeding of the IEEE Conference on Computer Vision and Pattern Recognition, 42 (2007) 1-7.

[19] J. Lie and M. Lysaker and X. C. Tai A Variant of the Level Set Method and Applications to Image Segmentation, Mathematics of Computation, 75, No. 1 (2006) 1155-1174.

[20] G. Murtaza and H. Ali and N. Badshah A Robust Local Model for Segmentation Based on Coefficient of Variation, Journal of Information and Communication Technology, 5, No. 1 (2011) 30-39.

[21] D. Mumford and J. Shah Optimal approximation by Piecewise Smooth Functions and Associated Variational Problems, Communications on Pure Applied Mathematics, 42 (1989) 577-685.

[22] L. A. Vese and T. F. Chan A Multiphase Level Set Framework for Image Segmentation using the Mumford and Shah model, International Journal of Computer Vision, 50, No. 3 (2002) 271-293.

[23] W. Wang and X. Wenyuan and W. Haibo and Suhua An Active Contour Model for Selective Segmentation, Acta Automatica Sinica, 2 (2005)36-49.

[24] X. F. Wang and D. S. Huang and H. Xu An Efficient Local Chan-Vese Model for Image Segmentation, Pattern Recognition, 43 (2010) 603-618.

[25] Y. Wu and C. He. Convex Variational Level Set Model for Image Segmentation, Signal Processing, 106 (2015)123-133.

[26] D. Xaiojun and D. B. Tien A New Model for Image Segmentation, IEEE Signal Processing Letters, 15(2008) 182-185.

[27] K. Zhang and H. Song and L. Zhang Active contours Driven by Local Binary Fitting Energy, Pattern Recognition, 43 (2010) 1199-1206.

[28] Z. Zhang and C.Duan and T. Lin and S. Zhou and Y. Wang and X. Gao A Novel External Force for Active Contour Based Image Segmentation, Information Sciences, 506 (2020) 1-18. 